\title{
Overview of DM searches
}

\section{Yeongduk Kim*†}

Inst. Basic Science, Korea

E-mail: ydkimeibs.re.kr

The identification of dark matter particles has been attempted for more than 30 years through various experimental methods. Theoretical studies are being performed to propose new particles as dark matter particle candidates. At present, we do not have a clear understanding about the nature of dark matter particles. In this brief review, I will describe the status and persepectives of the searches for each dark matter candidate.

35th International Cosmic Ray Conference

10-20 July, 2017

Bexco, Busan, Korea

*Speaker.

${ }^{\dagger}$ A footnote may follow. 


\section{Dark Matter Candidates}

There is a great puzzle about the universe. Astronomical observations, including the velocities of stars and galaxies and gravitational lensing measurements, have revealed that most of the universe is not made from the ordinary matter we are familiar with [1,2]. There is compelling evidence that $95 \%$ of the mass and energy in the universe is dark in the sense that it is not visible $[3,4]$. Further observational studies tell us that $26 \%$ of the dark component is in the form of clumps of dark matter and the remaining, $69 \%$ is homogeneous dark energy. The identification of dark matter and the elucidation of its properties is considered to be one of the most important issues in contemporary science because it will affect the basic understanding of the structure and origin of the universe and perhaps provide insights about particle physics beyond the standard model $[5,6]$. Many theoretical physicists consider that super-symmetric weakly interacting massive particles (WIMPs) are the most probable particle candidates for dark matter and that the extremely rarely occurring interactions of WIMPs with the nuclei of normal matter may be measured using a very low-background detector in a deep underground environment [7].

According to current theoretical research, there are a number of dark matter candidates in addition to WIMPs as shown in Fig. 1. The mass of dark matter particles ranges from $10^{-22}$ $\mathrm{eV}$ to $10^{23} \mathrm{eV}$ depending on their nature [8]. WIMPs have been leading candidates [7] and have been searched the most intensively; however there is no clear signal of WIMPs yet. Axions and axion-like particles (ALPs) are other strong candidates, and recently several different methods of searching these particles are being developed. [2, 10, 9] are good sources of reviews about dark matter.

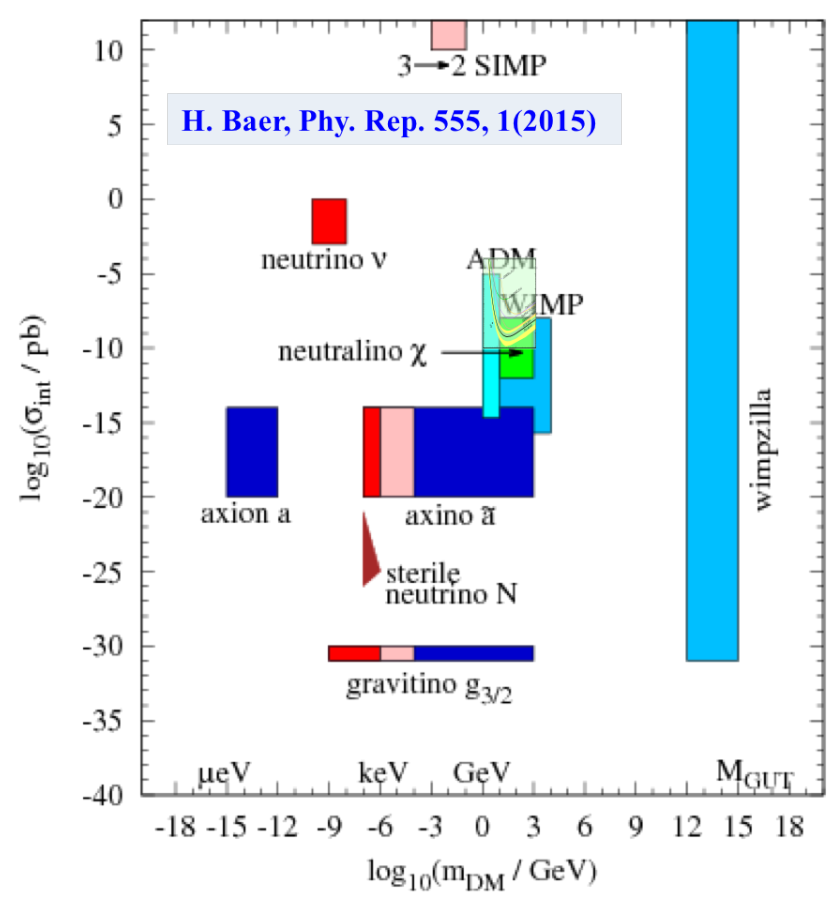

Figure 1: Particle candidates for dark matter. The figure is taken from [8]

Generally, dark matter particles may interact with or decay into standard model particles be- 
cause dark matter and visible matter are correlated in the distribution [11]. We can search for standard model particles after interaction with dark matter particles (direct search) or from dark matter annihilation or decay (indrect search) as shown schematically in Fig. 2. In addition, we can produce and identify dark matter particles through collisions of standard model particles using high energy particle accelerators. These three strategies to search for dark matter particles in nature can be applied for WIMPs and other dark matter particle candidates such as dark photons, ALPs, and sterile neutrinos. Only the direct search method has been applied for axions until now.

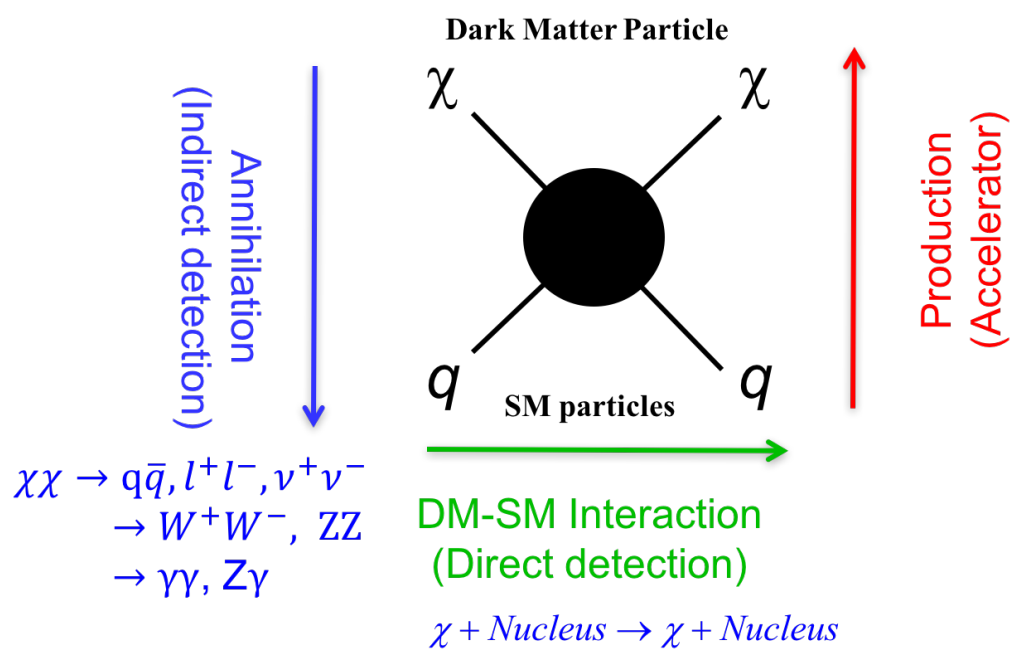

Figure 2: Mothods of searching for dark matter particles. Dark matter (DM) particles and standard model (SM) particles can interact via three different channels. DM decays can be considered using a slightly different diagram.

\section{WIMPs}

\subsection{WIMP Miracle}

WIMPs were first proposed as dark matter particles by B.W. Lee and Weinberg [7] in 1977. The particles considered were heavy neutrinos which interact weakly with hadrons, and annihilation rate was calculated as a function of freezing out temperature. The calculation exhibited remarkable consistency with the current dark matter energy density in the universe.

$$
\Omega_{D M} h^{2} \sim \frac{3 \times 10^{-27} \mathrm{~cm}^{3} \mathrm{~s}^{-1}}{\left\langle\sigma_{a n n} v\right\rangle}
$$

The estimated annihilation cross section was $\left\langle\sigma_{a n n} v\right\rangle \sim 3 \times 10^{-26} \mathrm{~cm}^{3} \mathrm{~s}^{-2}$, which gives $\Omega_{D M} \sim$ 0.22 . This consistency with the observed $26 \%$ of dark matter contents is referred to as the "miracle."

\subsection{Direct Searches}

Since Goodman and Witten proposed the possibility of searching for WIMPs through direct scattering of dark matter particles off nuclei [12], extensive programs are being performed to search 
for the WIMPs. One critical question is that "what kind of signal from WIMPs do we expect in direct detection experiments ?" There are four different signals expected from WIMP-nucleus interactions.

- Recoil energy spectrum as expected at low energy.

- Annual modulation due to the annual motion of the solar system.

- Directional asymmetry due to the daily rotation of Earth.

- $\mathrm{A}^{2}$ dependence (in the case of spin-independent interaction, $\mathrm{A}$ is the mass number of the target nucleus).

The differential elastic recoil event rate as a function of the recoil energy is expressed as [13] ;

$$
\begin{aligned}
\frac{d R\left(v_{E}, v_{e s c}\right)}{d E_{R}} & =k \frac{R_{0}}{E_{0} r}\left[c_{1} e^{-c_{2} E_{R} / E_{0} r}-e^{-v_{e s c}^{2} / v_{0}^{2}}\right] \\
R_{0} & =\frac{2 v_{0}}{\sqrt{\pi}} \frac{N_{A}}{A} \frac{\rho_{\chi}}{M_{\chi}} \sigma_{\chi A}^{S I(S D)} \\
\sigma_{\chi A}^{S I}=\frac{\mu_{A}^{2}}{\mu_{n}^{2}} A^{2} \sigma_{\chi n}^{S I} F_{S I}^{2}(q), \quad \sigma_{\chi A}^{S I} & =\frac{\mu_{A}^{2}}{\mu_{n}^{2}} \frac{4}{3} \frac{J+1}{J}\left(\left\langle S_{p}\right\rangle^{2} \sigma_{\chi p}^{S D}+\left\langle S_{n}\right\rangle^{2} \sigma_{\chi n}^{S D}\right) F_{S D}^{2}(q)
\end{aligned}
$$

Here, $v_{0}$ and $E_{0}=\frac{1}{2} M_{\chi} v_{0}^{2}$ is the most probable dark matter velocity and energy respectively, $v_{e s c}$ is the galactic dark matter particle escaping velocity, $v_{E}$ is the Earth velocity relative to dark matter distribution, A is the mass number of target nucleus, $N_{A}$ is Avogadro's number, $\rho_{\chi}$ is the dark matter local density, $M_{\chi}$ is the dark matter mass, $\sigma_{\chi A}^{S I(S D)}$ are the dark matter - nucleus spinindependent (SI) and spin-dependent (SD) cross section respectively. $k, c_{1}, c_{2}$ factors are calculated from the integration over the relative velocity distribution owing to the motion of the Earth over $v_{\text {min }}$ and $v_{\text {esc }}, \mu_{A}$ and $\mu_{n}$ are the reduced mass of dark matter particle and the nucleus (nucleon). $\sigma_{\chi n}^{S I}$ is the dark matter - nucleon spin-independent cross section, $\sigma_{\chi p(\chi n)}^{S D}$ is the dark matter - proton(neutron) spin-dependent cross section, J is the total spin of the target nucleus, $\left\langle S_{p(n)}\right\rangle^{2}$ are the amount of spin carried by the proton and neutron groups inside the nucleus, and $F_{S I(S D)}^{2}(q)$ are the form factor for spin-independent and spin-dependent interaction respectively [13].

\subsubsection{Direct Detection Techniques}

Recoil energies for the direct detection can be as large as $\sim 100 \mathrm{keV}$; thus they can be measured using conventional detectors. However, the backgrounds from environmental radioactivity significantly hinder the searches. As the backgrounds are mostly because of the interactions of beta and gamma rays with the detectors, techniques of differentiating these backgrounds from nuclear recoil (NR) signals have been developed. Particle interactions in detector material generates various signals which can be extracted using sensors, scintillation lights, vibrational phonons, and ionized charges. Multiple parameter detection techniques have been developed to differentiate NR signals from electron recoil (ER) signals. Figure 3 shows the various techniques and experiments of measuring multiple parameters. 
Double phase noble gas detectors are the most interesting developments for direct dark matter detection. They provide advantages in fiducial volume confinement and particle identification. Among detectors, noble gas liquids such as liquid xenon or liquid argon are used the most for dark matter experiments. Another multi-parameter detector, i.e., the SuperCDMS [14] experiment, measures ionization and phonons at low temperature conditions. The CRESST [15] detectors measure scintillation lights and phonons instead of ionization.

In addition, there are single-parameter detectors for direct dark matter detection. $\mathrm{NaI}(\mathrm{Tl})$ crystal experiments, i.e., DAMA, COSINE, and ANAIS, measure the scintillation lights in crystals while XMASS and DEAP measure scintillation lights from noble gases. The CDEX and CDMSlite experiments measure ionization only for lower detection thresholds in cryogenic detectors. the SuperCDMS group has developed HV detectors with only phonon sensors on each face and no ionization sensors to lower the threshold.

The DAMIC [16] and PICO [17] experiments detect the total energy deposited inside absorber material.

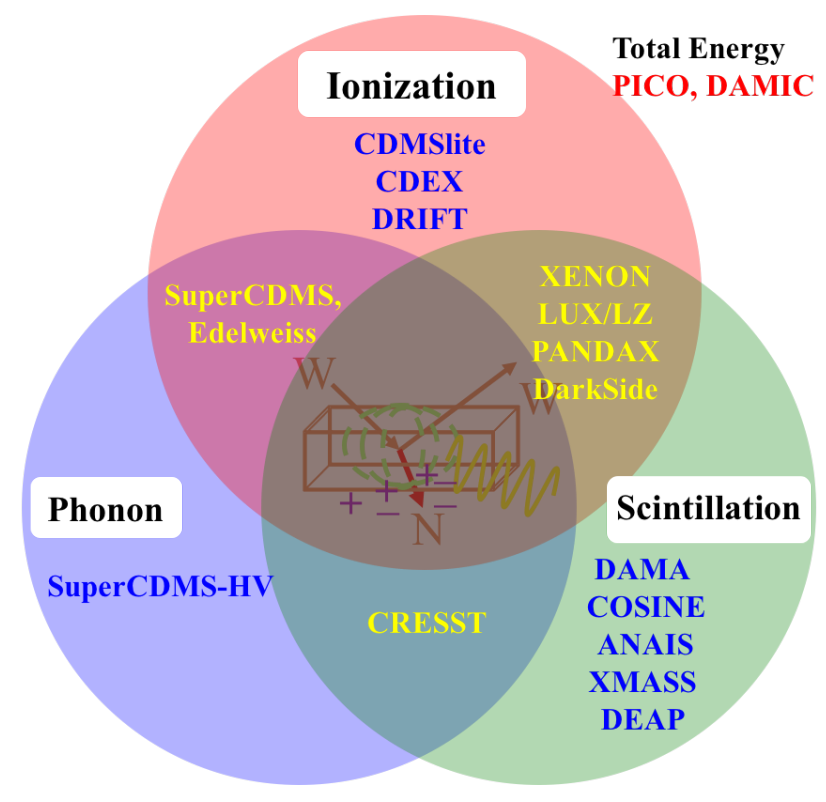

Figure 3: Techniques utilized by the direct dark matter search experiments. The three signals from dark matter interaction, scintillation, ionization, and phonon signals are measured in various experiments. Double parameter measurements are critical to differentiate nuclear recoil events from electron recoil backgrounds.

\subsubsection{Noble Gas Experiments}

Figure 4 shows the status of current double phase noble gas detectors, i.e., the LUX [23], PANDAX-II [24], XENON1T [25], and DarkSide-50 [21] experiments. The double phase experiments confine the fiducial volume for background rejection, and the fiducial mass used for dark matter detection is considerably smaller than the total mass used for the detectors. Exposure is obtained by multiplying fiducial mass by data collection time in units of, for example, $\mathrm{kg} \cdot$ year. The most recently reported data are from the XENON1T experiment and they show the largest exposure, $98 \mathrm{~kg}$ · year, among the double phase noble gas experiments. All double phase experiments 
reported basically zero background data inside the signal gate set by neutron and gamma source bands.

\begin{tabular}{ccccc}
\hline & & \\
\hline
\end{tabular}

Figure 4: Specifications of current double phase noble gas detectors. Exposure and Limit are the values reported in the most updated data for each experiments (LUX [23], PANDAX-II [24], XENON1T [25], and DarkSide-50 [21]).

The noble gas double phase experiments reported the lowest limits for the WIMP-nucleon spin-independent cross section achieved at certain WIMP masses in a range of $30-50 \mathrm{GeV}$. Figure 5 shows the lowest limits reported in all the experiments until now as a function of the maximum exposure reported by each experiment. As the backgrounds in the signal window are basically zero, the limits are proportional to $1 / \sqrt{\text { exposure }}$ as shown in the figure. The value of PANDAX-II is higher than expected limits by a factor of approximately two, and the reason for this is currently not clear.

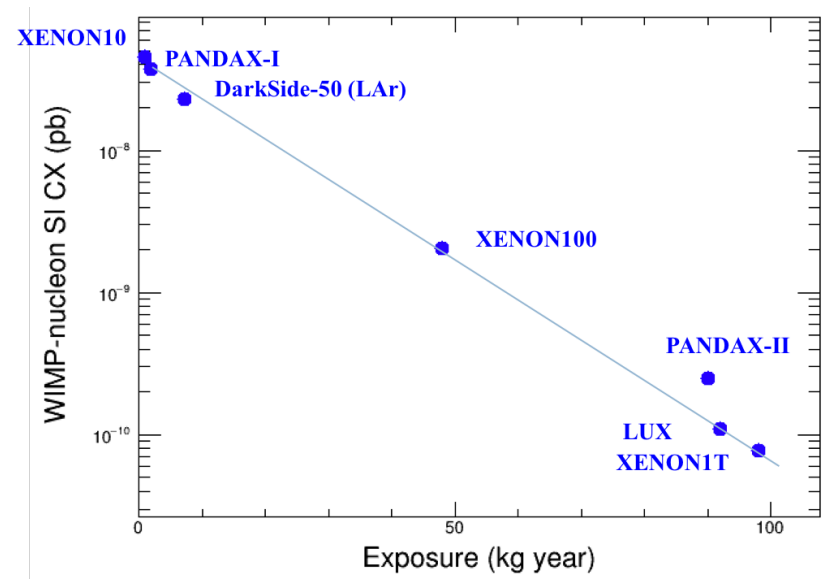

Figure 5: Lowest limits reported in each experiment as a function of the exposure for XENON10 [19] , PANDAX-I [20], DarkSide-50 [21] , XENON100 [22] , PANDAX-II [24], LUX [23], and XENON1T [25]. 
As the noble gas double phase detectors are the most successful for high mass WIMP search, larger scale experiments are proposed, as shown in Fig. 6 with the expected sensitivity for cross section limits. The LZ and XENONnT experiments are under development to be run around 2020. They could reach a cross section of $10^{-48} \mathrm{~cm}^{2}$. DARWIN [26] is the largest scale experiment with a fiducial mass of 30 tons; it can reach $10^{-49} \mathrm{~cm}^{2}$ hitting atmospheric neutrino backgrounds. As DARWIN will barely touch the neutrino backgrounds, at least one approximately 30-ton scale liquid xenon double phase experiment should be performed whether or not we detect some hints of WIMPs using the LZ or XENONnT detectors.

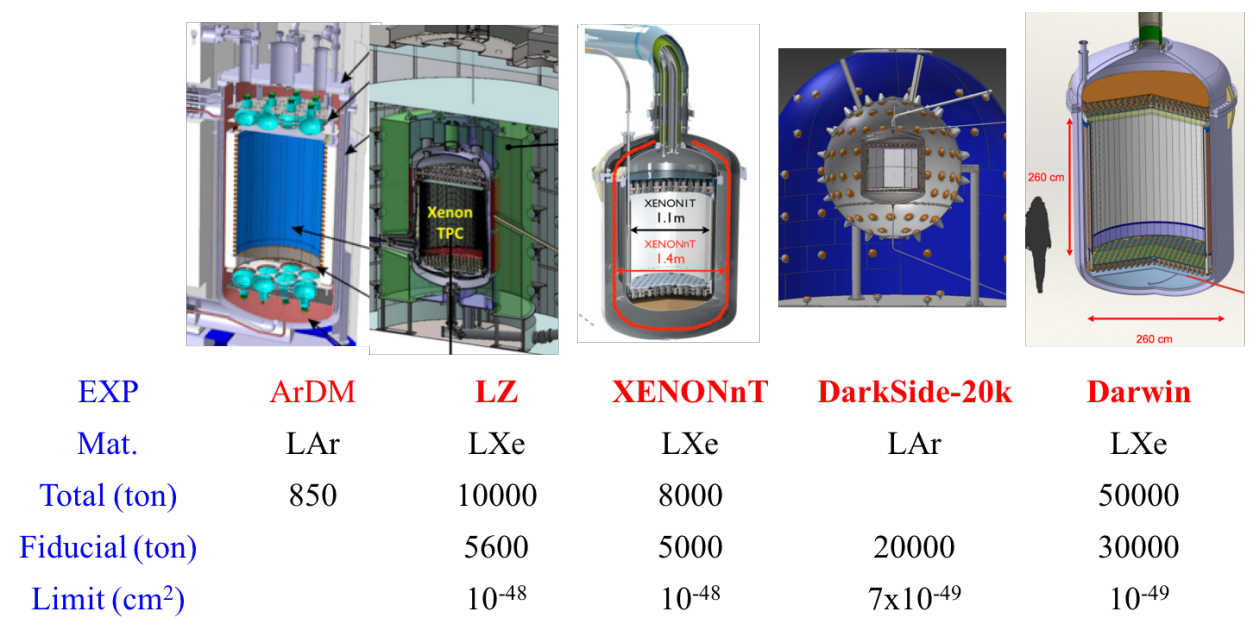

Figure 6: Proposed double phase noble gas experiments.

There are two single phase noble gas detectors running at present. The XMASS-I detector at the Kamioka laboratory consists of $832 \mathrm{~kg}$ of LXe filled as an active scintillation target with 642 hexagonal PMTs mounted on an inner spherical surface with a radius of approximately $40 \mathrm{~cm}$. A fiducial volume within a reconstructed radius of $20 \mathrm{~cm}$ is selected to reduce the event that is generated in the detector surface. $200 \mathrm{~kg}$ · year is the largest exposure with the fiducial volume confinement among noble gas detectors, and WIMP limits are provided based on the background spectrum in the fiducial volume [27].

DEAP-3600 is a single-phase liquid argon (LAr) detector with a capacity of 3600kg; it is installed $2 \mathrm{~km}$ underground at SNOLAB (Sudbury, Canada). The substantial difference between the LAr scintillation timing of NRs and ERs allows for sufficient rejection of the dominant $\beta, \gamma$ backgrounds using only primary scintillation light. The fiducial mass for the first analysis is 2.22 tons and the exposure is $27.0 \mathrm{~kg} \cdot$ year for only 4.44 days of live time data collection. No candidate signal events are observed, which results in the upper limit of the WIMP-nucleon spin-independent cross section as $1.2 \times 10^{44} \mathrm{~cm}^{2}$ for a WIMP mass of $100 \mathrm{GeV} / \mathrm{c}^{2}$ [28]. The initial data for DEAP3600 show potential upgrade to a larger mass detector for high mass WIMPs compatible with LXe double phase detectors.

\subsubsection{Cryogenic Experiments}

SuperCDMS SNOLAB is a next-generation experiment aimed at directly detecting low-mass dark matter particles (with masses $\leq 10 \mathrm{GeV} / \mathrm{c}^{2}$ ) using two types of cryogenic detectors ( $\mathrm{HV}$ and 
iZIP) and two target materials (germanium and silicon). The experiment is being designed with an initial sensitivity to NR cross sections of $\sim 1 \times 10^{43} \mathrm{~cm}^{2}$ for a dark matter particle mass of 1 $\mathrm{GeV} / \mathrm{c}^{2}$ and with a capacity to continue exploration of smaller masses and better sensitivities.

Two detector designs, denoted by HV and iZIP, have common physical dimensions (100 $\mathrm{mm}$ in diameter and $33.3 \mathrm{~mm}$ thick) and are fabricated from the same materials using the same techniques. Each $\mathrm{Ge}(\mathrm{Si})$ crystal has a mass of $1.39(0.61) \mathrm{kg}$. The HV detectors are designed to provide better sensitivity for mass $\leq 5 \mathrm{GeV} / \mathrm{c}^{2}$, while iZIPs provide better sensitivity above $\sim 5 \mathrm{GeV} / \mathrm{c}^{2}$ because of their capability to discriminate between ER and NR interactions. HV detectors consist of six phonon sensors on each face with no ionization sensors. The phonon-only sensor layout allows for better phonon collection and thus better phonon energy resolution and detector sensitivity at lower recoil energies compared to a similar iZIP detector. The expected sensitivity for the Ge HV setup is shown in Fig. 7.

The CRESST group is updating their low mass search experiment with scintillating crystals connected to transition-edge-sensors (TES). The CRESST-III detector module consists of a $24 \mathrm{~g}$ $\mathrm{CaWO}_{4}$ crystal $\left(20 \times 20 \times 10 \mathrm{~mm}^{3}\right)$ as target material, which is referred to as the phonon detector, and a silicon-on-sapphire (SOS) disc $\left(20 \times 20 \times 0.4 \mathrm{~mm}^{3}\right)$ as a light absorber which is referred to as the light detector [18]. The mass of the CRESST-III detector module is significantly lower than that of the CRESST-II crystals $(\sim 250 \mathrm{~g})$.

A prototype CRESST-III module has successfully been tested in a cryogenic setup above ground. An NR energy threshold of $\mathrm{E}_{\mathrm{th}}=(190.6 \pm 5.2) \mathrm{eV}$ was reached with the $24 \mathrm{~g}$ CaWO4 crystal. This is the lowest threshold reported for direct dark matter search experiments. The result demonstrates that the main design goal of CRESST-III phase 1, namely, a threshold of $\mathrm{E}_{\text {th }}=100 \mathrm{eV}$, should be easily achieved when the detectors are operated in the low-noise CRESST setup [18]. At present, 10 CRESST-III detector modules are assembled and mounted in the CRESST setup at the Gran Sasso laboratory. The expected sensitivity for CRESST (with $1000 \mathrm{~kg} \cdot$ day data) is shown in Fig. 7.

\subsubsection{Current Limits}

The plot of spin-indendent WIMP-nucleon cross sections versus WIMP mass is shown in Fig. 7. The solid lines represent experimental data and the dashed lines represent the expected limits of future experiments. The plot is produced using the WIMP limit plotter [29] by CDMS group. The SuperCDMS and CRESST experiments are leading the searches for low mass WIMPs below $10 \mathrm{GeV}$; however, approximately three orders of magnitudes are left over solar neutrino backgrounds. There were several claims that low mass WIMP signals may have been detected, including the DAMA/LIBRA, CoGENT [30], CRESST, and CDMS-Si experiments. Howevr, except DAMA/LIBRA, CRESST and CDMS groups confirmed that there is no signal persistent with more data, and the CoGENT experiment reported lower significance owing to the uncertainty in background estimation [30]. Therefore, at present, there is no strong indication for low mass WIMPs except the annual modulation data reported by the DAMA/LIBRA experiment.

\subsubsection{DAMA Experiment}

DAMA/LIBRA data clearly show annual modulation with an amplitude of $0.0112 \pm 0.0012$ counts/keV/kg/day (differential rate unit, dru) and a phase of $144 \pm 7$ days for 14 years of data 


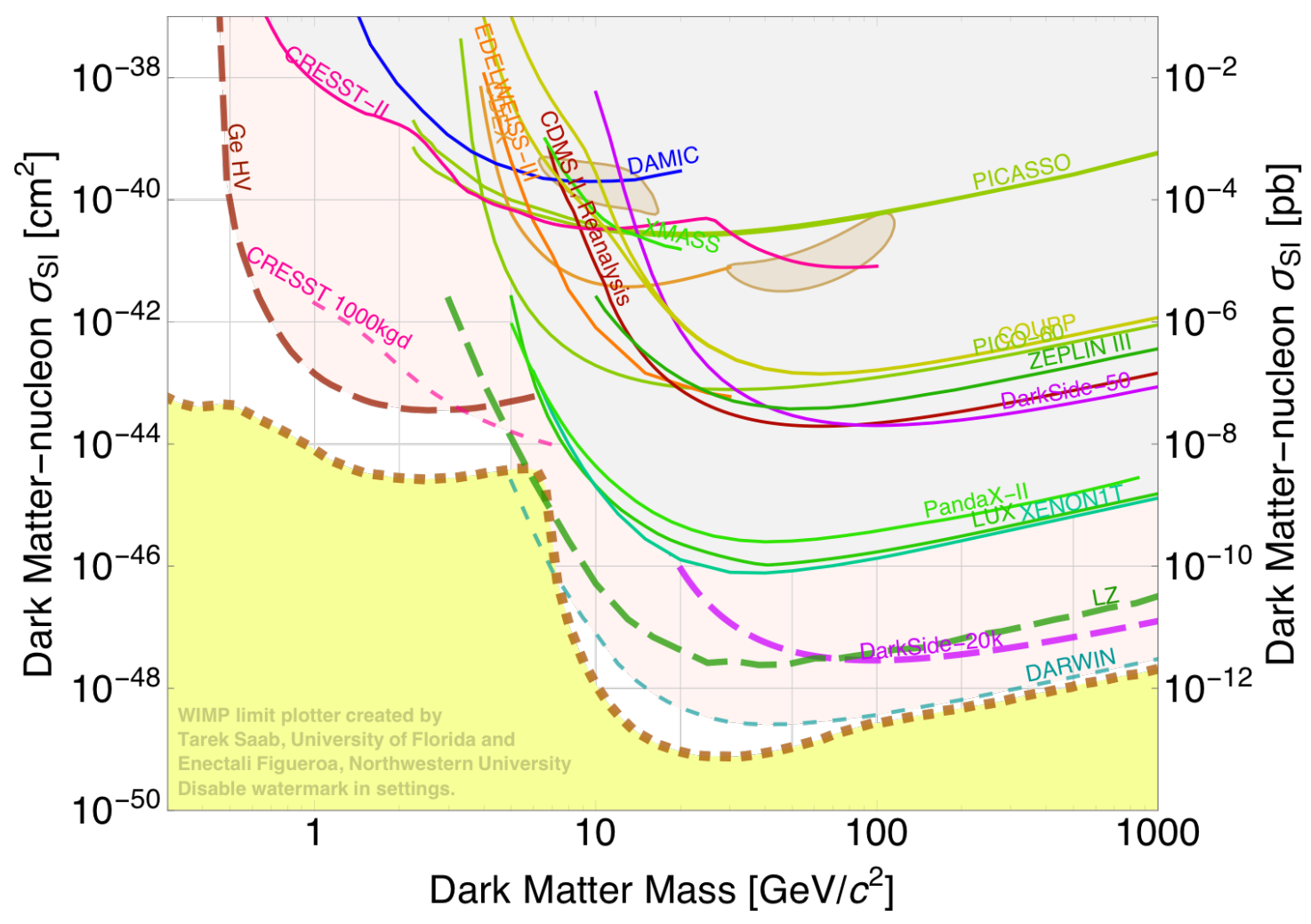

Figure 7: The spin-indendent WIMP-nucleon cross sections versus WIMP mass. The solid lines are experimental data and dashed lines are expected limits for future experiments. The plot is produced using WIMP limit plotter by CDMS group [29].

$[31,32]$. As muons and the neutrons induced by underground muons modulate in phases similar to the DAMA/LIBRA data, it is natural to speculate that the origin of DAMA/LIBRA data may be due to the cosmic muons. However, detailed simulation with underground muon flux shows that the flux of neutrons at the DAMA detector location is too low to explain the DAMA/LIBRA modulation amplitude $[37,33]$. In addition, the modulation phase of DAMA/LIBRA is faster by a month compared to the phases of the modulation by neutrons or muons measured at the Gran Sasso laboratory.

There was theoretical speculation that the phase difference between muon flux modulation and the DAMA modulation is owing to the contribution on the neutron flux from solar neutrinos [34]. As the phase of the modulations of solar neutrinos is opposite to that of atmospheric muons, a minor contribution of the neutrons from solar neutrino interactions with the surrounding materials of the DAMA detector could shift the phase by a month. However, this argument is not consistent with the more quantitative simulations with realistic experimental conditions. The neutrons from the neutrino interactions are extremely few [35], and the neutrons from deep underground cosmic muons are not sufficiently abundant [36, 37].

The analysis for potential annual modulation signals from several experiments has been reported. KIMS-CsI [38] provided an upper limit of 0.0119 dru with $90 \%$ confidence for the same 


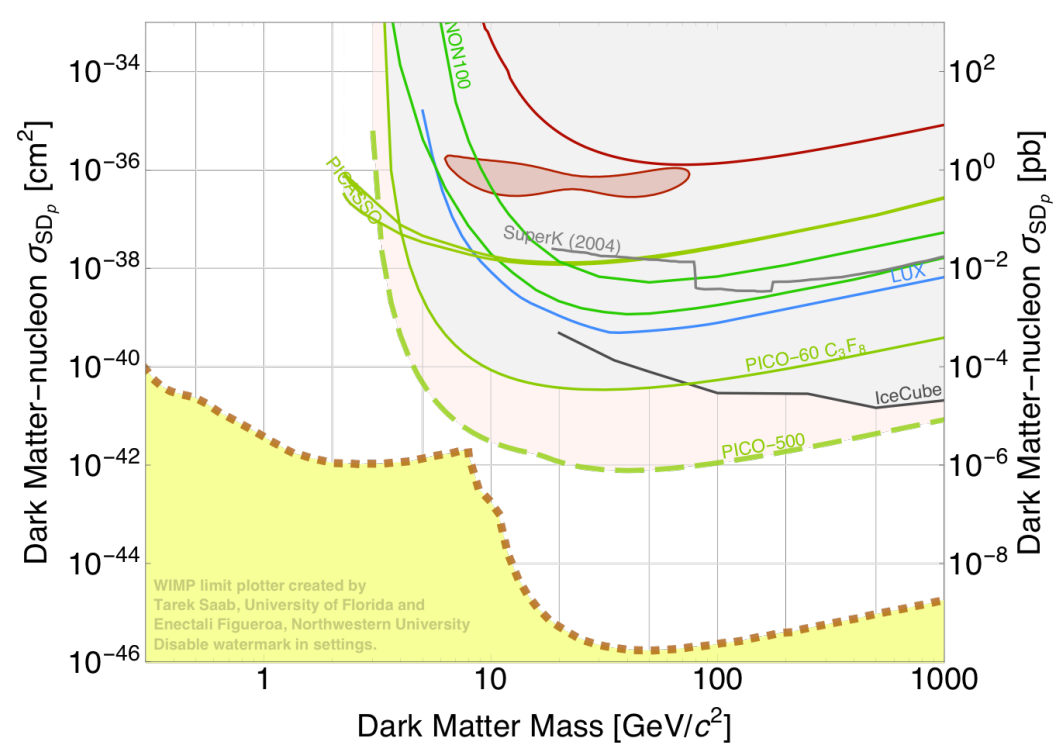

Figure 8: The spin-dendent WIMP-proton cross sections versus WIMP mass. The solid lines are experimental data and dashed lines are expected limits for future experiments. The plot is produced using WIMP limit plotter by CDMS group [29].

energy window of 2-6 keV as DAMA data. The XENON100 experiment [39] provided a modulation amplitude of $1.67 \pm 0.73$ events $/(\mathrm{keV} \cdot \mathrm{ton} \cdot$ day) compared to the expected amplitude of $12.2 \pm 1.2_{\text {stat }} \pm 0.7_{\text {syst }}$ events $/(\mathrm{keV} \cdot$ ton $\cdot$ day $)$, which is contradictory DAMA data with $5.7 \sigma$. The CDMS [40] experiment provided an upper limit of 0.06 events $/\left(\mathrm{keV}_{\mathrm{nr}} \cdot \mathrm{kg} \cdot\right.$ day $)$. In addition, the XMASS experiment provided results on annual modulation [41] with an upper limit of $(1.7 \sim 3.7) \times 10^{3}$ events $/$ day $/ \mathrm{kg} / \mathrm{keV}_{\text {ee }}$. Even though most direct dark matter experiments are contradictory to the modulation amplitude measured by the DAMA group, there are theoretical models that attempt to explain the DAMA modulation signals and null results from other experiments simultaneously through WIMP interaction in the DAMA/LIBRA detectors [42, 43, 44] .

The following four experimental groups are attempting to directly (de)confirm directly DAMA/LIBRA data using the same NaI(Tl) crystals; SABRE [46], ANAIS [47], PICO-LON [48], and COSINE [45] experiments. Saint-Gobain Crystals, which developed and produced the DAMA/LIBRA crystals, no longer produces the ultra-low backgrond $\mathrm{NaI}(\mathrm{Tl})$ crystals. Therefore, new experiments should perform R\&D from the beginning with other companies to develop ultra-low background $\mathrm{NaI}(\mathrm{Tl})$ crystals. It would require several years to develop crystals with a quality similar to that of the DAMA/LIBRA crystals. The ANAIS and COSINE experiments achieved a background level of approximately 2-5 dru below the $6 \mathrm{keV}$ energy region. The ANAIS group began an experiment with $112 \mathrm{~kg}$ crystals in the summer of 2017 and COSINE group began $110 \mathrm{~kg}$ crystal experiment in the Fall of 2016. The DAMA group has collected approximately 6 more years of data using the new Hamamatsu PMTs and will release the data in the near future. It is interesting to compare the DAMA/LIBRA data and the data from the COSINE and ANAIS experiments.

\subsubsection{New Techniques}

While liquid xenon detectors are being rapidly developed and the upper limits on the WIMP- 
nucleon cross section in the higher WIMP mass region are being reduced, there are considerable efforts toward further investigation of low mass WIMP search. If we use liquid xenon as a superheated material rather than $\mathrm{CF}_{3} \mathrm{I}$, we can observe scintillation in addition to the bubbles from NR events. The Scintillating Bubble Chamber has been demonstrated [49].

Target nuclei should be light to be sensitive to low mass WIMPs below $10 \mathrm{GeV}$. Helium is one of the best candidates, and the double phase liquid helium detector is interesting [50]. Another proposals using liquid helium for WIMP search are appeared [51, ?]. Inside liquid helium, elementary excitations (phonons or rotons) are produced owing to the WIMP-nucleus interaction. Excitations which have an energy greater than the binding energy of helium to the surface can result in the evaporation of helium atoms. It was proposed to detect these atoms by ionizing them in a strong electric field. Because the binding energy of helium to surfaces can be below $1 \mathrm{meV}$, this detection scheme opens up new possibilities for the detection of dark matter particles in a mass range down to $1 \mathrm{MeV} / \mathrm{c}^{2}[52]$.

A prototype $0.5 \mathrm{~g}$ sapphire detector developed by CRESST group has achieved an energy threshold of $19.7 \pm 0.9 \mathrm{eV}$. This low threshold allows to probe elastic scattering of dark matter particles down to masses of $140 \mathrm{MeV} / \mathrm{c}^{2}$ [53]. The next generation of gram-scale calorimeters which will be developed for the $v$-cleus experiment may extend the reach of dark matter particle masses of $\mathrm{O}\left(10 \mathrm{MeV} / \mathrm{c}^{2}\right)$.

Several ideas about new detectors particularly for low mass dark matter particles were proposed in a recent workshop, "US Cosmic Visions : New Ideas in Dark Matter 2017 [54]."

\subsection{Indirect Searches}

The flux of standard model particles from WIMP annhilation, as shown in Fig. 2 is the product of three factors, i.e. the number of WIMPs, the annihilation cross section, and the number of standard model particles per annihilation. The differential standard model particle flux from the WIMP annhilation can be expressed as ;

$$
\frac{d \varphi}{d \Omega d E}=\frac{\left\langle\sigma v_{\text {rel }}\right\rangle}{8 \pi m_{\chi}^{2}} \times \frac{d N_{S M}}{d E} \times \int_{\text {l.o.s. }} d s \rho(\vec{r}[s, \Omega])^{2}
$$

Here, $\int_{\text {l.o.s. }} d s \rho(\vec{r}[s, \Omega])^{2} / m_{\chi}^{2}$ is the number of dark matter particles in the solid angle of line of sight of measurement, $\frac{d N_{S M}}{d E}$ is the differential number of standard model particles from each dark matter annihilation, and $\left\langle\sigma v_{\text {rel }}\right\rangle$ is the annihilation cross section of dark matter particles. For the annihilation cross section, $\left\langle\sigma v_{\text {rel }}\right\rangle \sim 3 \times 10^{-26} \mathrm{~cm}^{3}$ is a good miraculous benchmark value as described in section 2.1 and it does not depend on WIMP mass [2]

One of the main challenges that are encountered when searching for signatures from dark matter annihilation in the cosmic gamma-ray flux is the discrimination between a possible signal and the astrophysical background.

\subsubsection{Diffuse Gamma-ray Excess}

There were several claims about an excess of gamma rays approximately $1 \mathrm{GeV}$ from the galactic center of our galaxy, the Milky Way [55]. This could be because approximately $35 \mathrm{GeV}$ WIMPs annihilated at a rate of $\left\langle\sigma v_{\text {rel }}\right\rangle \sim 1.7 \times 10^{-26} \mathrm{~cm}^{3} / \mathrm{s}$. However, recent measurements could 
not confirm the excess gamma rays as due to the WIMP annihilations. Even though the galactic center gamma-ray excess is present over estimated backgrounds, other sources such as milisecond pulsars can produce similar excess [56]. Therefore, a dark matter interpretation of the galactic center excess cannot be robustly claimed and upper limits are provided.

Figure 9 shows the $90 \%$ confidence limits for the annihilation rate obtained through various indirect search experiments, reproduced from a reference by Conrad [59]. The Icecube and ANTARES data are from neutrino measurements, the AMS data are from $e^{+} e^{-}$pair measurements, and Planck is the cosmic microwave background measurement. Generally, the neurino channels in the $10-1000 \mathrm{GeV}$ mass region are less sensitive to other channels. The indication of WIMP annihilation reported from Fermi-LAT data is represented by the region circled in red [55] . The expected sensitivity of the Cherenkov Telescope Array (CTA) experiment is added to the original figure and shown with a dashed line [58].

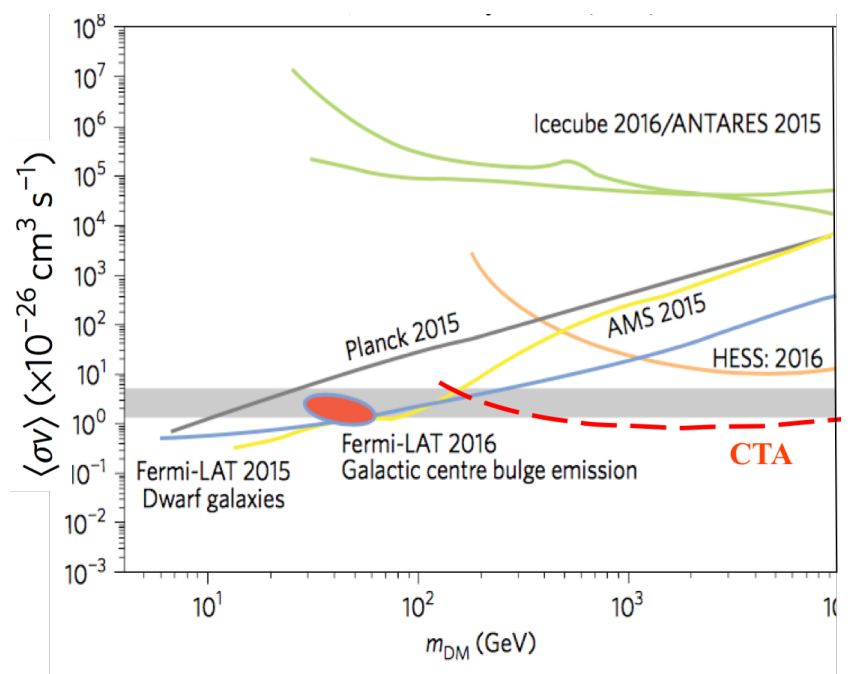

Figure 9: WIMP annihilation rate limits from indirect search experiments reproduced from ref. [59]. Refer to [59] for the details of each experiment and [58] for the expected sensitivity of CTA experiment.

\subsubsection{Line Gammas}

WIMP pairs can annihilate into a photon $(\gamma)$ and a second particle (X); for example, $\gamma \gamma, \gamma Z$, or $\gamma \mathrm{H}$. As dark matter is electrically neutral and has no direct coupling to photons, the $\chi \chi \rightarrow \gamma X$ process is suppressed. Photons are monochromatic with an energy of

$$
E_{\gamma}=m_{X}\left(1-\frac{m_{X}^{2}}{4 m_{X}^{2}}\right)
$$

Detections of a linelike feature at $130 \mathrm{GeV}$ have been reported in literature. This feature is reported to be strongly correlated with the Galactic center region [60, 61] However, recent data from HESS [62] and Fermi-LAT [63] show no enhancements in the energy region of $130 \mathrm{GeV}$.

\subsubsection{Decaying Heavy DM search}

Another channel that has been studied for dark matter annihilation is neutrinos. Neutrinos from Earth, the Sun, and galactic and extra-galactic sources are measured to find excesses in the energy 
region of the detectors [64]. The ICE-CUBE and ANTARES detectors discovered that ultra-high energy neutrinos up to $10^{16} \mathrm{eV}$ can be measured, and the direction appeared to be isotropic [66] The origin of this high energy neutrino flux with purely atmospheric contribution being disfavoured at over $5 \sigma$ remains unidentified. As decaying heavy dark matter particles are predicted in several models, the observation of high-energy neutrinos enables one to probe heavy decaying dark matter at the corresponding mass scales. Fits are performed on experimental data. The background hypothesis is the atmospheric flux and diffuse astrophysical flux; the signal hypothesis additionally allows for flux from decaying dark matter. Even though the fits in the analyses converge to a finite dark matter lifetime, they are consistent with background fluctuations [67]

\subsubsection{Charged Particles}

Since the Alpha Magnetic Spectrometer (AMS) group first announced their positron and antiproton data $[68,69,70]$, there have been numerous theoretical models that explain the data based on dark matter annihilations[71, 72]. However, there are also multiple theoretical explanations based on astrophysical sources such as nearby supernovas and pulsars [73, 74]. A recent ovewview of AMS data and theoretical interpretations is presented by A, Kounine [75] in this conference.

Cosmic rays and their secondary backgrounds are highly energetic up to $10^{21} \mathrm{eV}[76,77]$. In addition, their rates are several orders higher than the expected dark matter indirect signals for the similar energy region. Furthermore, the origin of the cosmic-ray particle acceleration mechanism is not clearly understood yet. Therefore, large uncertainties typically exist in the estimation of the backgrounds for indirect dark matter signals. To improve the certainty about the origin of the flux of antiparticles, more precise estimation of the backgrounds with more detailed simulations is required. The estimation of systematic uncertainties is critical to extract small potential signals.

\section{Axions and Axion-Like Particles}

Axions are hypothetical particles associated with the spontaneous breaking of the PecceiQuinn symmetry [79]. Axions are light, and axion mass is given as

$$
m_{a} \sim \frac{\Lambda_{Q C D}^{2}}{f_{a}} \sim 0.6 e V\left(\frac{10^{7} \mathrm{GeV}}{f_{a}}\right)
$$

Here, $f_{a}$ is the axion decay constant, and axion mass ranges from $10^{-12} \mathrm{eV} \leq m_{a} \leq 1 \mathrm{MeV}$ for $f_{a}$ to be between $100 \mathrm{GeV}$ and the Planck scale [79].

Axions are frequently considered as an alternative to WIMPs and have recently received considerable experimental attention. Axions can be searched experimentally by (1) searching for the decay of axions in astrophysical objects, (2) producing and detecting axions in laboratories ("shinethrough-wall" experiments), (3) detecting galactic axions using the microwave cavities, and (4) searching for axions produced in the Sun or nuclear reactors, which are strong gamma-ray sources. Dark matter axions can be searched using axion-photon conversion in the presence of EM fields (Primakoff effect) [85].

$$
g_{a \gamma} \sim\left(1.3 \times 10^{-15} / \mathrm{GeV}\right) \frac{m_{a}}{10^{-5} \mathrm{eV}}
$$


The interaction strength of axions is determined by their mass, as mentioned above; however ALPs can have independent coupling and mass.

\subsection{Solar Axion Searches with Dark Matter Detectors}

There are two well-known axion models, i.e. the KSVZ (hadronic) [80, 81] and DFSZ (nonhadronic) $[82,83]$ models. The strengths of axion-photon $\left(g_{a \gamma}\right)$, axion-electron $\left(g_{a e}\right)$ and axionnucleon $\left(g_{a N}\right)$ couplings are different for both models. In particular, the axion-electron coupling in the DFSZ model occurs at the tree level while the axion-electron coupling in the KSVZ model is strongly suppressed owing to axion-electron coupling at the loop level. Thus, in the DFSZ model, the processes related to axion-electron coupling prevail over the Primakoff process, with axion-photon coupling as an axion production mechanism in stars and the Sun: Compton scattering $(\gamma+\mathrm{e} \rightarrow \mathrm{e}+\mathrm{a})$, axio-recombination $(\mathrm{e}+\mathrm{A} \rightarrow \mathrm{A}+\mathrm{a})$, axio-deexcitation $(\mathrm{A} \rightarrow \mathrm{A}+\mathrm{a})$, axiobremsstrahlung $(\mathrm{e}+\mathrm{A} \rightarrow \mathrm{e}+\mathrm{A}+\mathrm{a})$, and electron-electron collision $(e+e \rightarrow e+e+a)$, where $\mathrm{A}$ is an atom [89]. The total axion flux produced on Earth from the Sun was recently estimated in ref. [84], which includes processes with axion-electron and axion-photon couplings, as shown in Fig. 10.

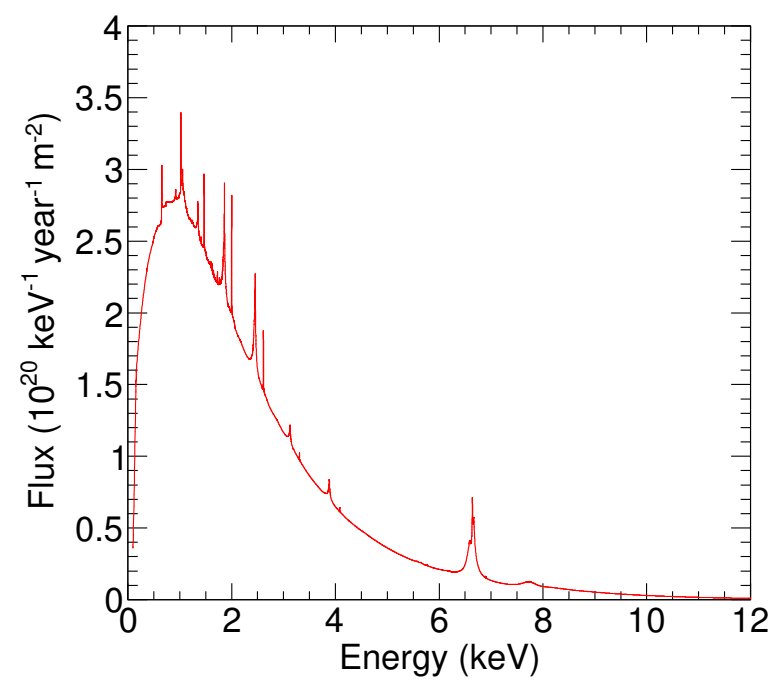

Figure 10: Flux of solar axions due to Compton scattering, axio-recombination, axio-deexcitation, axiobremsstrahlung and electron-electron collisions on Earth [84] with an axion-electron coupling of $g_{a e}=10^{13}$. The figure is from [89]

The cross section for the axio-electric effect is given by

$$
\sigma_{a e}\left(E_{a}\right)=\sigma_{p e}\left(E_{a}\right) \frac{g_{a e}^{2}}{\beta_{a}} \frac{3 E_{a}^{2}}{16 \pi \alpha m_{e}^{2}}\left(1-\frac{\beta_{a}^{2 / 3}}{3}\right),
$$

where $\mathrm{E}_{\mathrm{a}}$ is axion energy, $\sigma_{p e}$ is the photoelectric cross section for target nuclei, $g_{a e}$ is axionelectron coupling, $\beta_{a}$ is the axion velocity over the speed of light, $\alpha$ is the fine structure constant, and $\mathrm{m}_{\mathrm{e}}$ is electron mass. 
Using the measured background specrum for dark matter experiments, one can deduce the upper limits for the cross section of each energy bin and for the $g_{a e}$ parameter between solar axions and electrons. The LUX, XMASS, XENON100, KIMS-CsI experiments have provided the upper limits [86, 87, 88, 89] as shown in Fig. 11.

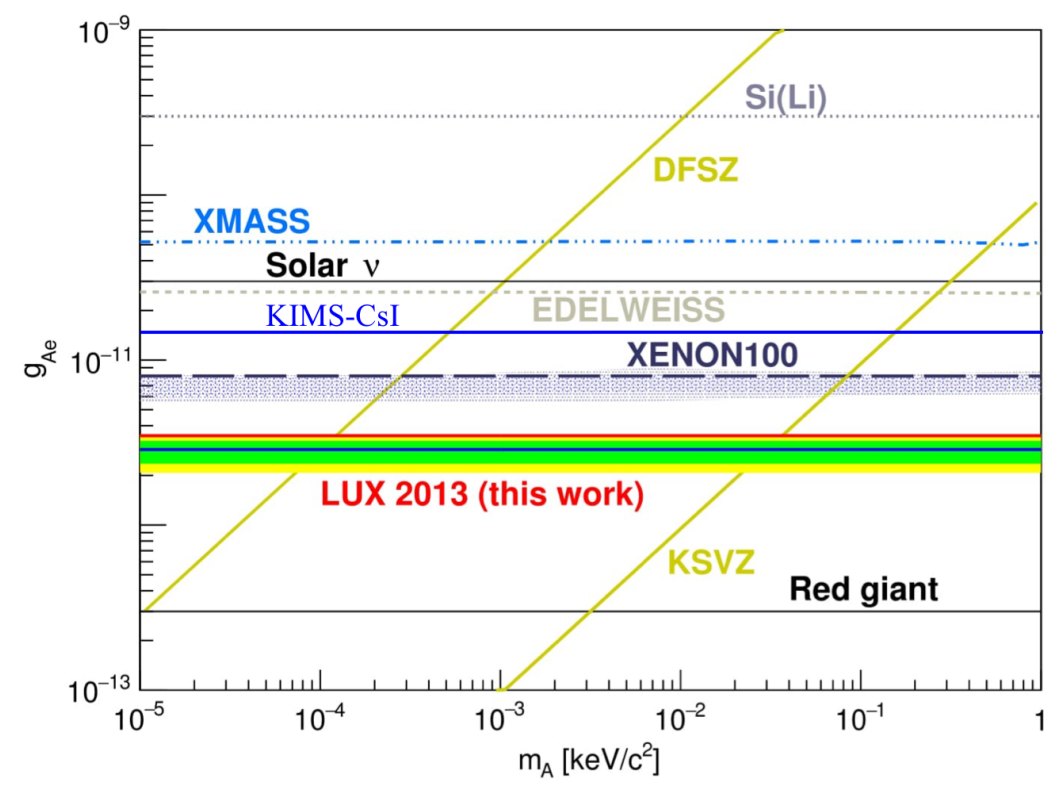

Figure 11: Red curve: LUX 2013 data; 90\% C.L. limit on the coupling between solar axions and electrons. Blue curve: $90 \%$ C.L. sensitivity, $\pm 1 \sigma$ (green band), and $\pm 2 \sigma$ (yellow band).

\subsection{Axion-Like Particle Searches}

If ALPs constitute a significant fraction of cold dark matter, then they could be detected through their coupling to photons in magnetic fields. Photon-ALP interactions could leave an imprint on $\gamma$-ray spectra, provided that ALP mass is sufficiently small $\left(\mathrm{m}_{\mathrm{ALP}} \leq \mu \mathrm{eV}\right.$ [90]). The Fermi-LAT group searched for spectral irregularities in the $\gamma$-ray spectrum of NGC 1275, which is the central galaxy of the Perseus cluster. Using 6 years of Fermi-LAT data, they found no evidence for ALPs and excluded couplings above $5 \times 10^{12} \mathrm{GeV}^{1}$ for ALP masses of $0.5 \leq \mathrm{m}_{\mathrm{ALP}} \leq 5 \mathrm{neV}$ at $95 \%$ confidence [90].

\section{Sterile Neutrinos}

Sterile neutrinos are good candidates for warm dark matter if their mass is at the $\mathrm{keV}$ scale. The LSND experiment [91] indicated eV scale sterile neutrinos, and the reactor neutrino anomaly [92] was also interpreted owing to the eV scale sterile neutrinos. However, recent results for short baseline neutrino oscillation experiments (NEOS [93]) show no indication of eV scale sterile neutrinos. Furthermore Daya Bay data indicate that the reactor neutrino anomaly is due to the uncertainties in the theoretical estimation of antineutrino flux from ${ }^{235} \mathrm{U}$ [94]. In addition, ICECUBE and MINOS data exclude the $\sim \mathrm{eV}$ parameter region of LSND data as neutrino oscillations [95, 96]. There- 


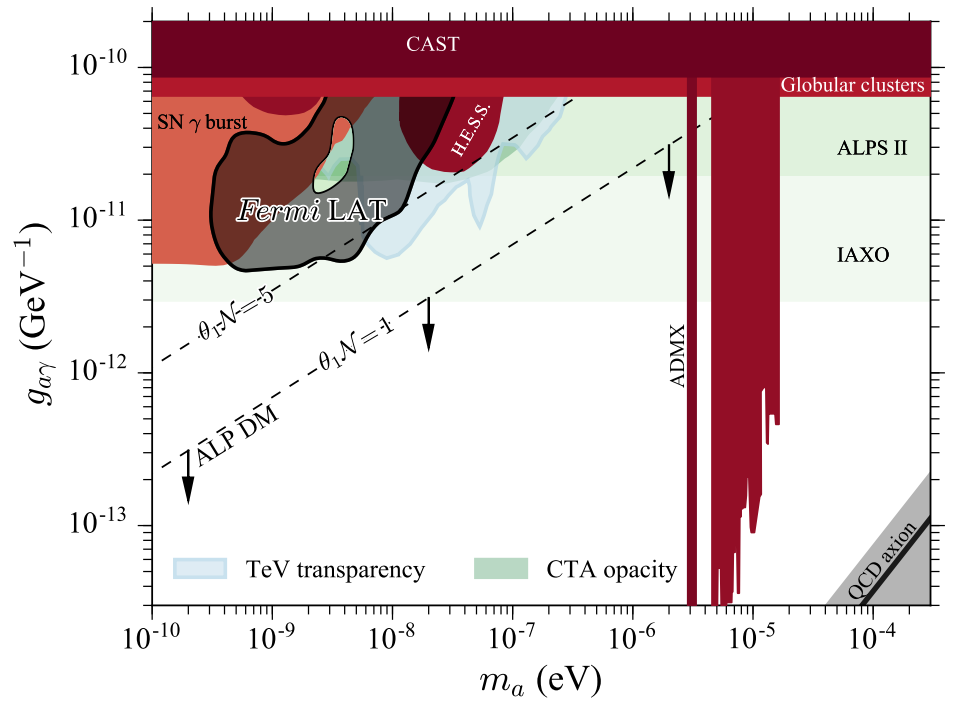

Figure 12: Comparison of Fermi-LAT limits with other works. Other limits are shown in red, expected sensitivities in green. The parameter space where ALPs could explain a low $r m \gamma-$ ray opacity is shown in blue. The QCD axion is shown as a gray shaded band and solid black line. The figure is taken from Fig. 2 in [90]

fore, the eV scale sterile neutrino indications are becoming weaker; however more experimental investigations are required.

If sterile neutrinos decay to active neutrinos with a long halflife $\left(v_{s} \rightarrow v_{a}+\gamma\right)$, then the energy of gammas are $E_{\gamma}=m_{s} / 2$. There were claims that the X-ray detection in space by the XMMNewton observatory shows enhancement in the $3.5 \mathrm{keV}$ energy region [97]. This can be interpreted as the decay of $7 \mathrm{keV}$ sterile neutrinos, which can form the warm dark matter halo. High-resolution X-ray spectroscopy with Hitomi was expected to resolve the origin of the faint unidentified $\mathrm{E} \sim$ $3.5 \mathrm{keV}$ emission line reported in several low-resolution studies of various massive systems such as galaxies and clusters, including the Perseus cluster [98]. Unfortunately, the Hitomi spacecraft lost its ground contact on 26 March 2016, and the recovery operation by JAXA was subsequently discontinued. Even though data were collected for a short period, no $3.5 \mathrm{keV}$ peak was observed and data were inconsistent with XMM-Newton data [98].

\section{Summary}

Dark matter candidates become more diverse from $10^{-22} \mathrm{eV} \sim 10^{25} \mathrm{eV}$, including fuzzy dark matter, dark photon, asymmetric dark matter, and strongly interacting dark matter etc. We must search for dark matter more broadly because there is no preferable candidate at present. For WIMPs, the high mass region will be searched with larger LXe or LAr detectors, at a scale of approximately 50 tons. The lower mass region requires a lower threshold, and several new techniques are being developed. The DAMA conumdrum should be solved in the near future. Indirect searches will be further developed for higher mass WIMPs and improved sensitivity. Further back- 
ground understanding is critical! More diverse interpretation for various candidates will be pursued further for ALPs and dark photons. "Normal Science" based on the $\Lambda$ CDM paradigm should be continuously pursued to find more "real" anomalies to develop new physics.

At present, the standard model works extremely well with almost no anomaly. There appear to be several anomalies; however they should be confirmed as facts and should be proved to be inconsistent with all interpretations from the existing paradigm. An anomaly can be produced through rigorous verification processes. Exploring more parameter spaces using new techniques and experiments should be pursued until real anomalies are confirmed. Thomas Kuhn stated the following: "Anomaly appears only against the background provided by the paradigm. The more precise and far-reaching that paradigm is, the more sensitive an indicator it provides of anomaly and hence of an occasion for paradigm change [99]." The backgrounds in direct and indirect dark matter searches are obstacles for the searches; however they are also a playground searching for a potential new anomaly. 


\section{References}

[1] D. Clowe, M. Bradac, A. H. Gonzalez, M. Markevitch, S. W. Randall, C. Jones and D. Zaritsky, A direct empirical proof of the existence of dark matter Astrophys. J. 648, L109 (2006) [astro-ph/0608407].

[2] Stefano Profumo, An Introduction to Particle Dark Matter' World Scientific (2016)

[3] G. Hinshaw et al. [WMAP Collaboration], Nine-Year Wilkinson Microwave Anisotropy Probe (WMAP) Observations: Cosmological Parameter Results Astrophys. J. Suppl. 208, 19 (2013) [arXiv:1212.5226 [astro-ph.CO]].

[4] P. A. R. Ade et al. [Planck Collaboration], Planck 2015 results. XIII. Cosmological parameters Astron. Astrophys. 594, A13 (2016) [arXiv:1502.01589 [astro-ph.CO]].

[5] G. Bertone, D. Hooper and J. Silk, Particle dark matter: Evidence, candidates and constraints Phys. Rept. 405, 279 (2005) [hep-ph/0404175].

[6] L. Bergstrom, Dark Matter Candidates New J. Phys. 11, 105006 (2009) [arXiv:0903.4849 [hep-ph]].

[7] B. W. Lee and S. Weinberg, Cosmological Lower Bound on Heavy Neutrino Masses Phys. Rev. Lett. 39, 165 (1977).

[8] H. Baer, K. Y. Choi, J. E. Kim and L. Roszkowski, Dark matter production in the early Universe: beyond the thermal WIMP paradigm Phys. Rept. 555, 1 (2015) [arXiv:1407.0017 [hep-ph]].

[9] J. L. Feng, Dark Matter Candidates from Particle Physics and Methods of Detection Ann. Rev. Astron. Astrophys. 48, 495 (2010) [arXiv:1003.0904 [astro-ph.CO]].

[10] T. MarrodÃąn Undagoitia and L. Rauch, Dark matter direct-detection experiments J. Phys. G 43, no. 1, 013001 (2016) [arXiv:1509.08767 [physics.ins-det]].

[11] R. Massey et al., Dark matter maps reveal cosmic scaffolding Nature 445, 286 (2007) [astro-ph/0701594].

[12] M. W. Goodman and E. Witten, Detectability of Certain Dark Matter Candidates Phys. Rev. D 31, 3059 (1985).

[13] J. D. Lewin and P. F. Smith, Astropart. Phys. 6, 87 (1996). doi:10.1016/S0927-6505(96)00047-3

[14] R. Agnese et al. [SuperCDMS Collaboration], Projected Sensitivity of the SuperCDMS SNOLAB experiment Phys. Rev. D 95, no. 8, 082002 (2017) [arXiv:1610.00006 [physics.ins-det]].

[15] G. Angloher et al. [CRESST Collaboration], Results on light dark matter particles with a low-threshold CRESST-II detector Eur. Phys. J. C 76, no. 1, 25 (2016) [arXiv:1509.01515 [astro-ph.CO]].

[16] A. Aguilar-Arevalo et al. [DAMIC Collaboration], First Direct-Detection Constraints on eV-Scale Hidden-Photon Dark Matter with DAMIC at SNOLAB Phys. Rev. Lett. 118, no. 14, 141803 (2017) [arXiv:1611.03066 [astro-ph.CO]].

[17] C. Amole et al. [PICO Collaboration], Dark Matter Search Results from the PICO-60 $\mathrm{C}_{3} \mathrm{~F}_{8}$ Bubble Chamber Phys. Rev. Lett. 118, no. 25, 251301 (2017) [arXiv:1702.07666 [astro-ph.CO]].

[18] R. Strauss et al., A prototype detector for the CRESST-III low-mass dark matter search Nucl. Instrum. Meth. A 845, 414 (2017). 
[19] J. Angle et al. [XENON Collaboration], First Results from the XENON10 Dark Matter Experiment at the Gran Sasso National Laboratory Phys. Rev. Lett. 100, 021303 (2008) [arXiv:0706.0039 [astro-ph]].

[20] M. Xiao et al. [PandaX Collaboration], First dark matter search results from the PandaX-I experiment Sci. China Phys. Mech. Astron. 57, 2024 (2014) [arXiv:1408.5114 [hep-ex]].

[21] P. Agnes et al. [DarkSide Collaboration], Results from the first use of low radioactivity argon in a dark matter search Phys. Rev. D 93, no. 8, 081101 (2016) Addendum: [Phys. Rev. D 95, no. 6, 069901 (2017)] [arXiv:1510.00702 [astro-ph.CO]].

[22] E. Aprile et al. [XENON100 Collaboration], XENON100 Dark Matter Results from a Combination of 477 Live Days Phys. Rev. D 94, no. 12, 122001 (2016) [arXiv:1609.06154 [astro-ph.CO]].

[23] D. S. Akerib et al. [LUX Collaboration], Results from a search for dark matter in the complete LUX exposure Phys. Rev. Lett. 118, no. 2, 021303 (2017) [arXiv:1608.07648 [astro-ph.CO]].

[24] A. Tan et al. [PandaX-II Collaboration], Dark Matter Results from First 98.7 Days of Data from the PandaX-II Experiment Phys. Rev. Lett. 117, no. 12, 121303 (2016) [arXiv:1607.07400 [hep-ex]].

[25] E. Aprile et al. [XENON Collaboration], First Dark Matter Search Results from the XENON1T Experiment arXiv:1705.06655 [astro-ph.CO].

[26] J. Aalbers et al. [DARWIN Collaboration], DARWIN: towards the ultimate dark matter detector JCAP 1611, 017 (2016) [arXiv:1606.07001 [astro-ph.IM]].

[27] H. Ogawa for XMASS collaboration, ICRC2017 proceedings.

[28] P.-A. Amaudruz et al. [DEAP-3600 Collaboration], First results from the DEAP-3600 dark matter search with argon at SNOLAB arXiv:1707.08042 [astro-ph.CO].

[29] http://cdms.berkeley.edu/limitplots/mm/WIMP_limit_plotter.html.

[30] C. E. Aalseth et al. [CoGeNT Collaboration], Search for An Annual Modulation in Three Years of CoGeNT Dark Matter Detector Data arXiv:1401.3295 [astro-ph.CO].

[31] R. Bernabei et al. [DAMA Collaboration], First results from DAMA/LIBRA and the combined results with DAMA/NaI Eur. Phys. J. C 56, 333 (2008) [arXiv:0804.2741 [astro-ph]].

[32] R. Bernabei et al., Final model independent result of DAMA/LIBRA-phase1 Eur. Phys. J. C 73, 2648 (2013) [arXiv:1308.5109 [astro-ph.GA]].

[33] R. Bernabei et al., No role for muons in the DAMA annual modulation results Eur. Phys. J. C 72, 2064 (2012) [arXiv:1202.4179 [astro-ph.GA]].

[34] J. H. Davis, Fitting the annual modulation in DAMA with neutrons from muons and neutrinos Phys. Rev. Lett. 113, 081302 (2014) [arXiv:1407.1052 [hep-ph]].

[35] P. S. Barbeau, J. I. Collar, Y. Efremenko and K. Scholberg, Comment on "Fitting the annual modulation in DAMA with neutrons from muons and neutrinos” Phys. Rev. Lett. 113, no. 22, 229001 (2014) [arXiv:1409.3185 [hep-ph]].

[36] J. Klinger and V. A. Kudryavtsev, Muon-induced neutrons do not explain the DAMA data Phys. Rev. Lett. 114, no. 15, 151301 (2015) [arXiv:1503.07225 [hep-ph]].

[37] E. Jeon and Y. Kim, A simulation-based study of the neutron backgrounds for NaI dark matter experiments Astropart. Phys. 73, 28 (2016) [arXiv:1503.07918 [hep-ex]].

[38] Presentation by Y.D. Kim at "Closing in on Dark Matter”, Aspen, Jan. 28 - Feb. 3 (2013) 
[39] E. Aprile et al. [XENON Collaboration], Search for Electronic Recoil Event Rate Modulation with 4 Years of XENON100 Data Phys. Rev. Lett. 118, no. 10, 101101 (2017) [arXiv:1701.00769 [astro-ph.CO]].

[40] Z. Ahmed et al. [CDMS-II Collaboration], Search for annual modulation in low-energy CDMS-II data arXiv:1203.1309 [astro-ph.CO].

[41] K. Abe et al. [XMASS Collaboration], Direct dark matter search by annual modulation in XMASS-I Phys. Lett. B 759, 272 (2016) [arXiv:1511.04807 [astro-ph.CO]].

[42] S. Scopel and K. H. Yoon, JCAP 1602, no. 02, 050 (2016) doi:10.1088/1475-7516/2016/02/050 [arXiv:1512.00593 [astro-ph.CO]].

[43] R. Cerulli, P. Villar, F. Cappella, R. Bernabei, P. Belli, A. Incicchitti, A. Addazi and Z. Berezhiani, DAMA annual modulation and mirror Dark Matter Eur. Phys. J. C 77, no. 2, 83 (2017) [arXiv:1701.08590 [hep-ex]].

[44] B. M. Roberts, V. A. Dzuba, V. V. Flambaum, M. Pospelov and Y. V. Stadnik, Dark matter scattering on electrons: Accurate calculations of atomic excitations and implications for the DAMA signal Phys. Rev. D 93, no. 11, 115037 (2016) [arXiv:1604.04559 [hep-ph]].

[45] G. Adhikari et al., Understanding NaI(Tl) crystal background for dark matter searches Eur. Phys. J. C 77, no. 7, 437 (2017) [arXiv:1703.01982 [astro-ph.IM]].

[46] C. Tomei [SABRE Collaboration], SABRE: Dark matter annual modulation detection in the northern and southern hemispheres Nucl. Instrum. Meth. A 845, 418 (2017).

[47] J. Amare et al., Assessment of backgrounds of the ANAIS experiment for dark matter direct detection Eur. Phys. J. C 76, no. 8, 429 (2016) [arXiv:1604.05587 [astro-ph.IM]].

[48] K. I. Fushimi et al., Search for cosmic dark matter by means of ultra high purity NaI(Tl) scintillator arXiv:1705.00110 [physics.ins-det].

[49] D. Baxter et al., First Demonstration of a Scintillating Xenon Bubble Chamber for Detecting Dark Matter and Coherent Elastic Neutrino-Nucleus Scattering Phys. Rev. Lett. 118, no. 23, 231301 (2017) [arXiv:1702.08861 [physics.ins-det]].

[50] W. Guo and D. N. McKinsey, Concept for a dark matter detector using liquid helium-4 Phys. Rev. D 87, no. 11, 115001 (2013) [arXiv:1302.0534 [astro-ph.IM]].

[51] K. Schutz and K. M. Zurek, Detectability of Light Dark Matter with Superfluid Helium Phys. Rev. Lett. 117, no. 12, 121302 (2016) [arXiv:1604.08206 [hep-ph]].

[52] H. J. Maris, G. M. Seidel and D. Stein, Dark Matter Detection Using Helium Evaporation and Field Ionization arXiv:1706.00117 [astro-ph.IM].

[53] G. Angloher et al. [CRESST Collaboration], Results on MeV-scale dark matter from a gram-scale cryogenic calorimeter operated above ground Eur. Phys. J. C 77, no. 9, 637 (2017) [arXiv:1707.06749 [astro-ph.CO]].

[54] M. Battaglieri et al., US Cosmic Visions: New Ideas in Dark Matter 2017: Community Report arXiv:1707.04591 [hep-ph].

[55] T. Daylan, D. P. Finkbeiner, D. Hooper, T. Linden, S. K. N. Portillo, N. L. Rodd and T. R. Slatyer, The characterization of the gamma-ray signal from the central Milky Way: A case for annihilating dark matter Phys. Dark Univ. 12, 1 (2016) [arXiv:1402.6703 [astro-ph.HE]]. 
[56] M. Ackermann et al. [Fermi-LAT Collaboration], The Fermi Galactic Center GeV Excess and Implications for Dark Matter Astrophys. J. 840, no. 1, 43 (2017) [arXiv:1704.03910 [astro-ph.HE]].

[57] H. Abdallah et al. [H.E.S.S. Collaboration], Search for dark matter annihilations towards the inner Galactic halo from 10 years of observations with H.E.S.S Phys. Rev. Lett. 117, no. 11, 111301 (2016) [arXiv:1607.08142 [astro-ph.HE]].

[58] J. Carr et al. [CTA Collaboration], PoS ICRC 2015, 1203 (2016) [arXiv:1508.06128 [astro-ph.HE]].

[59] J. Conrad and O. Reimer, Indirect dark matter searches in gamma and cosmic rays Nature Phys. 13, no. 3, 224 (2017) [arXiv:1705.11165 [astro-ph.HE]].

[60] T. Bringmann, X. Huang, A. Ibarra, S. Vogl and C. Weniger, Fermi LAT Search for Internal Bremsstrahlung Signatures from Dark Matter Annihilation JCAP 1207, 054 (2012) [arXiv:1203.1312 [hep-ph]].

[61] C. Weniger, A Tentative Gamma-Ray Line from Dark Matter Annihilation at the Fermi Large Area Telescope JCAP 1208, 007 (2012) [arXiv:1204.2797 [hep-ph]].

[62] H. Abdalla et al. [H.E.S.S. Collaboration], H.E.S.S. Limits on Linelike Dark Matter Signatures in the $100 \mathrm{GeV}$ to $2 \mathrm{TeV}$ Energy Range Close to the Galactic Center Phys. Rev. Lett. 117, no. 15, 151302 (2016) [arXiv:1609.08091 [astro-ph.HE]].

[63] M. Ackermann et al. [Fermi-LAT Collaboration], Search for Gamma-ray Spectral Lines with the Fermi Large Area Telescope and Dark Matter Implications Phys. Rev. D 88, 082002 (2013) [arXiv:1305.5597 [astro-ph.HE]].

[64] M. G. Aartsen et al. [IceCube Collaboration], Search for Neutrinos from Dark Matter Self-Annihilations in the center of the Milky Way with 3 years of IceCube/DeepCore arXiv:1705.08103 [hep-ex].

[65] M. G. Aartsen et al. [IceCube Collaboration], Observation and Characterization of a Cosmic Muon Neutrino Flux from the Northern Hemisphere using six years of IceCube data Astrophys. J. 833, no. 1, 3 (2016) [arXiv:1607.08006 [astro-ph.HE]].

[66] M. G. Aartsen et al. [IceCube Collaboration], All-sky Search for Time-integrated Neutrino Emission from Astrophysical Sources with 7 yr of IceCube Data Astrophys. J. 835, no. 2, 151 (2017) [arXiv:1609.04981 [astro-ph.HE]].

[67] Jöran Stettner Search for Signatures of Heavy Decaying Dark Matter with IceCube in proceedings of ICRC conference, POS ( ICRC2017) 923.

[68] M. Aguilar et al. [AMS Collaboration], First Result from the Alpha Magnetic Spectrometer on the International Space Station: Precision Measurement of the Positron Fraction in Primary Cosmic Rays of $0.5 \hat{a} \breve{A S} 350$ GeV Phys. Rev. Lett. 110, 141102 (2013).

[69] L. Accardo et al. [AMS Collaboration], High Statistics Measurement of the Positron Fraction in Primary Cosmic Rays of $0.5 \hat{a} \breve{A} 5500 \mathrm{GeV}$ with the Alpha Magnetic Spectrometer on the International Space Station Phys. Rev. Lett. 113, 121101 (2014).

[70] M. Aguilar et al. [AMS Collaboration], Antiproton Flux, Antiproton-to-Proton Flux Ratio, and Properties of Elementary Particle Fluxes in Primary Cosmic Rays Measured with the Alpha Magnetic Spectrometer on the International Space Station Phys. Rev. Lett. 117, no. 9, 091103 (2016).

[71] A. Ibarra, A. S. Lamperstorfer and J. Silk, Dark matter annihilations and decays after the AMS-02 positron measurements Phys. Rev. D 89, no. 6, 063539 (2014) [arXiv:1309.2570 [hep-ph]]. 
[72] L. Bergstrom, T. Bringmann, I. Cholis, D. Hooper and C. Weniger, New limits on dark matter annihilation from AMS cosmic ray positron data Phys. Rev. Lett. 111, 171101 (2013) [arXiv:1306.3983 [astro-ph.HE]].

[73] K. Kohri, K. Ioka, Y. Fujita and R. Yamazaki, Can we explain AMS-02 antiproton and positron excesses simultaneously by nearby supernovae without pulsars or dark matter? PTEP 2016, no. 2, $021 \mathrm{E} 01$ (2016) [arXiv:1505.01236 [astro-ph.HE]].

[74] M. Di Mauro, F. Donato, N. Fornengo, R. Lineros and A. Vittino, Interpretation of AMS-02 electrons and positrons data JCAP 1404, 006 (2014) [arXiv:1402.0321 [astro-ph.HE]].

[75] A. Kounine Properties of Elementary Particle Fluxes in Primary Cosmic Rays Measured with the Alpha Magnetic Spectrometer on the ISS in proceedings of ICRC conference, POS ( ICRC2017) 1093.

[76] Elisa Resconi High Energy Multi-Messenger Astronomy in proceedings of ICRC conference, POS ( ICRC2017).

[77] Luke Drury Galactic Cosmic Rays (Direct) Theory and Interpretation in proceedings of ICRC conference, POS ( ICRC2017).

[78] P. Mertsch and S. Sarkar, AMS-02 data confront acceleration of cosmic ray secondaries in nearby sources Phys. Rev. D 90, 061301 (2014) [arXiv:1402.0855 [astro-ph.HE]].

[79] J. E. Kim and G. Carosi, Axions and the Strong CP Problem Rev. Mod. Phys. 82, 557 (2010) [arXiv:0807.3125 [hep-ph]].

[80] J. E. Kim, Weak Interaction Singlet and Strong CP Invariance Phys. Rev. Lett. 43, 103 (1979).

[81] M. A. Shifman, A. I. Vainshtein and V. I. Zakharov, Can Confinement Ensure Natural CP Invariance of Strong Interactions? Nucl. Phys. B 166, 493 (1980).

[82] A. R. Zhitnitsky, On Possible Suppression of the Axion Hadron Interactions. (In Russian) Sov. J. Nucl. Phys. 31, 260 (1980) [Yad. Fiz. 31, 497 (1980)].

[83] M. Dine, W. Fischler and M. Srednicki, A Simple Solution to the Strong CP Problem with a Harmless Axion Phys. Lett. 104B, 199 (1981).

[84] J. Redondo, Solar axion flux from the axion-electron coupling JCAP 1312, 008 (2013) [arXiv:1310.0823 [hep-ph]].

[85] P. Sikivie, Experimental Tests of the Invisible Axion Phys. Rev. Lett. 51, 1415 (1983) Erratum: [Phys. Rev. Lett. 52, 695 (1984)].

[86] D. S. Akerib et al. [LUX Collaboration], First Searches for Axions and Axionlike Particles with the LUX Experiment Phys. Rev. Lett. 118, no. 26, 261301 (2017) [arXiv:1704.02297 [astro-ph.CO]].

[87] K. Abe et al., Search for solar axions in XMASS, a large liquid-xenon detector Phys. Lett. B 724, 46 (2013) [arXiv:1212.6153 [astro-ph.CO]].

[88] E. Aprile et al. [XENON100 Collaboration], First Axion Results from the XENON100 Experiment Phys. Rev. D 90, no. 6, 062009 (2014) Erratum: [Phys. Rev. D 95, no. 2, 029904 (2017)] [arXiv:1404.1455 [astro-ph.CO]].

[89] Y. S. Yoon et al. [KIMS Collaboration], Search for solar axions with CsI(Tl) crystal detectors JHEP 1606, 011 (2016) [arXiv:1604.01825 [hep-ex]]. 
[90] M. Ajello et al. [Fermi-LAT Collaboration], Search for Spectral Irregularities due to Photonâ̆ŞAxionlike-Particle Oscillations with the Fermi Large Area Telescope Phys. Rev. Lett. 116, no. 16, 161101 (2016) [arXiv:1603.06978 [astro-ph.HE]].

[91] C. Athanassopoulos et al. [LSND Collaboration], Evidence for $n u(m u) \longrightarrow$ nu(e) neutrino oscillations from LSND Phys. Rev. Lett. 81, 1774 (1998) [nucl-ex/9709006].

[92] G. Mention, M. Fechner, T. Lasserre, T. A. Mueller, D. Lhuillier, M. Cribier and A. Letourneau, Phys. Rev. D 83, 073006 (2011) doi:10.1103/PhysRevD.83.073006 [arXiv:1101.2755 [hep-ex]].

[93] Y. J. Ko et al., Sterile Neutrino Search at the NEOS Experiment Phys. Rev. Lett. 118, no. 12, 121802 (2017) [arXiv:1610.05134 [hep-ex]].

[94] F. P. An et al. [Daya Bay Collaboration], Evolution of the Reactor Antineutrino Flux and Spectrum at Daya Bay Phys. Rev. Lett. 118, no. 25, 251801 (2017) [arXiv:1704.01082 [hep-ex]].

[95] M. G. Aartsen et al. [IceCube Collaboration], Searches for Sterile Neutrinos with the IceCube Detector Phys. Rev. Lett. 117, no. 7, 071801 (2016) [arXiv:1605.01990 [hep-ex]].

[96] P. Adamson et al. [MINOS Collaboration], Search for Sterile Neutrinos Mixing with Muon Neutrinos in MINOS Phys. Rev. Lett. 117, no. 15, 151803 (2016) [arXiv:1607.01176 [hep-ex]].

[97] E. Bulbul, M. Markevitch, A. Foster, R. K. Smith, M. Loewenstein and S. W. Randall, Detection of An Unidentified Emission Line in the Stacked X-ray spectrum of Galaxy Clusters Astrophys. J. 789, 13 (2014) [arXiv:1402.2301 [astro-ph.CO]].

[98] F. A. Aharonian et al. [Hitomi Collaboration], Hitomi constraints on the $3.5 \mathrm{keV}$ line in the Perseus galaxy cluster Astrophys. J. 837, no. 1, L15 (2017) [arXiv:1607.07420 [astro-ph.HE]].

[99] Thomas Samuel Kuhn, The Structure of Scientific Revolution The University of Chicago, (2012) 